\title{
Evaluation of dog semen quality after slow (biological freezer) or rapid (nitrogen vapours) freezing
}

\author{
Ada RotA ${ }^{\mathrm{a} *}$, Alessandra RotA $^{\mathrm{b}}$, Marco MARTINI $^{\mathrm{c}}$, Chiara MILANI ${ }^{\mathrm{d}}$, \\ Stephano ROMAGNOLI ${ }^{\mathrm{d}}$ \\ a Department of Animal Pathology, Faculty of Veterinary Medicine, University of Turin, \\ via Leonardo da Vinci 44, Grugliasco (TC), Italy \\ $\mathrm{b}$ Department of Veterinary Clinics, Faculty of Veterinary Medicine, University of Pisa, \\ S. Piero a Grado (PI), Italy \\ ${ }^{c}$ Department of Public Health, Comparative Pathology and Veterinary Hygiene, \\ Faculty of Veterinary Medicine, University of Padua, Agripolis 35020 Legnaro (PD), Italy \\ ${ }^{d}$ Department of Veterinary Clinical Sciences, Faculty of Veterinary Medicine, \\ University of Padua, Agripolis 35020 Legnaro (PD), Italy
}

(Received 13 April 2004; accepted 1 October 2004)

\begin{abstract}
Three ejaculates were collected from each of five dogs. After initial evaluation, the spermrich fractions were diluted to $100 \times 10^{6}$ spermatozoa $\mathrm{mL}^{-1}$ in two steps with an egg yolk-TRIS extender containing a final concentration of 5\% glycerol and $0.5 \%$ Equex STM paste. Half of the $0.5 \mathrm{~mL}$ straws obtained from each ejaculate were frozen on nitrogen vapours $(4 \mathrm{~cm}$ above the liquid surface) ("rapid freezing"), while the other half was frozen in a biological freezer at a rate of $0.5^{\circ} \mathrm{C} \cdot \mathrm{min}^{-1}$ between $5^{\circ} \mathrm{C}$ and $-10{ }^{\circ} \mathrm{C}$ and of $8{ }^{\circ} \mathrm{C} \cdot \mathrm{min}^{-1}$ between $-10{ }^{\circ} \mathrm{C}$ and $-60{ }^{\circ} \mathrm{C}$, followed by immersion in liquid nitrogen ("slow freezing"). After an average storage of 30 days, the straws were thawed in a water-bath at $37^{\circ} \mathrm{C}$ for $1 \mathrm{~min}$. Progressive motility was subjectively estimated hourly for $8 \mathrm{~h}$ on semen incubated at $38^{\circ} \mathrm{C}$. Immediately after thawing and after $2 \mathrm{~h}$ of incubation, motility parameters were also measured by a motility analyser. Sperm membrane function and chromatin stability were assessed immediately post-thaw, using the hypo-osmotic swelling test and acridine orange staining, respectively. Slow freezing significantly improved total post-thaw motility, which showed a slower decline over time, although spermatozoal average path and straight line velocity were lower compared to the fast rate. Also the number of intact membrane spermatozoa was significantly higher in slow-frozen samples while the proportion of spermatozoa with single-stranded DNA was minimal after both freezing procedures.
\end{abstract}

$\mathrm{dog} /$ semen / cryopreservation / freezing rate

\section{INTRODUCTION}

Dog semen is most commonly frozen over nitrogen vapours, which is a practical and economical method when dealing with low numbers of straws. The results might be improved through a more controlled rate of temperature decrease. The critical steps of freezing procedures are the initial cooling rate, between the temperature at which the sample is collected and $+4{ }^{\circ} \mathrm{C}$, and the step when ice forms. Intracellular ice formation

* Corresponding author: ada.rota@ unito.it 
occurs when cells are rapidly cooled since not enough water is allowed to leave the cells which might be mechanically damaged by ice crystals. Damage can also occur if cooling is too slow, when dehydration is too severe and spermatozoa are exposed to a high solute concentration [1-3]. A freezing procedure is effective when an equilibrium between cooling and freezing rates and cryoprotectant concentration is found.

Cryodamages affect sperm survival and semen quality at thawing. Freezing of spermatozoa can result in damages that can be immediately recognised, or can produce alterations (i.e. to plasma membranes), which may have a delayed effect and result in a reduced sperm life-span $[4,5]$. The decreased post-thaw longevity is considered to be a critical factor for the fertility of cryopreserved dog spermatozoa. In vitro incubation of frozen-thawed spermatozoa at body temperature can partially simulate in vivo conditions and this test can be used to assess their longevity $[4,6]$. Cryodamages impairing sperm membrane function can be assessed by the Hypo-Osmotic Swelling (HOS) test, which consists in challenging spermatozoa with a hypo-osmotic solution to evaluate whether membranes are able to modulate water permeability and reach an equilibrium. The hypo-osmotic test has been used on many species and can be considered a simple analysis which could be added to routine semen evaluation [7]. The functional integrity of the sperm membrane is highly correlated to in-vitro fertilizing ability of spermatozoa in man [8] but not in bovine [9]. A correlation with motility was found in bovine spermatozoa [10] and with progressive motility in the stallion [7]. The HOS test has been used to assess the quality of both fresh [11] and frozen-thawed canine semen [12].

In man, DNA structure has been shown to be modified by the freezing-thawing process [13]; DNA integrity can be assessed with Acridin Orange (AO), a fluorochrome which fluoresces green when bound to normal DNA and orange/red when bound to single-stranded DNA [14]. The integrity of mammalian sperm chromatin is related to fertility [15] and a specific flow cytometric assay (sperm chromatin structure assay) has been developed and applied to several animal species such as the bull, stallion, boar [16] and cat [17]. A simplified method, not requiring flow cytometric equipment, was introduced by Tejada et al. [14].

The aim of this study was to compare the effects of two freezing procedures - a slow cooling rate obtained using a biological freezer and a rapid cooling rate obtained by placing the straws over nitrogen vapours on post-thaw motility parameters, plasma membrane function and DNA integrity of canine spermatozoa.

\section{MATERIALS AND METHODS}

The sperm-rich fraction of three ejaculates was collected by digital manipulation from each of 5 dogs of proven fertility, in the presence of a bitch in oestrus. All the dogs were clinically healthy at the time of collection and their age ranged between 1 and 7 years. Three dogs were crossbred, one was an English Setter and the fifth a Border Collie.

Motility in raw semen was immediately assessed subjectively under light microscopy at $38{ }^{\circ} \mathrm{C}$ and $400 \times$ magnification by two operators and spermatozoal concentration was determined using a haemocytometer (Bürker chamber). A motility above $80 \%$ and a total number of spermatozoa higher than $200 \times 10^{6}$ were fixed as minimum parameters for an ejaculate to be included in the experiment.

Semen was evaluated, centrifuged at $700 \mathrm{~g}$ for $5 \mathrm{~min}$ and the sperm pellet was resuspended in two steps with an egg yolk, TRIS, glycerol extender [4]. Spermatozoa were equilibrated at $4{ }^{\circ} \mathrm{C}$ for $1 \mathrm{~h}$, diluted in a first extender containing $3 \%$ glycerol. Thereafter an equal volume of the second extender (also at $4{ }^{\circ} \mathrm{C}$ ), containing $7 \%$ glycerol and $1 \%$ Equex STM paste (Nova Chemicals Sales, Scituate, MA, USA) was added, to 
obtain a final concentration of $5 \%$ glycerol, $0.5 \%$ Equex STM paste and $100 \times 10^{6}$ spermatozoa $\cdot \mathrm{mL}^{-1}$. After a further $10 \mathrm{~min}$ at $4{ }^{\circ} \mathrm{C}$, $0.5 \mathrm{~mL}$ straws were filled with the diluted semen and sealed with Seal-Ease (Becton Dickinson and Company, Franklin Lakes, NJ, USA). Half of the straws obtained from each ejaculate were laid horizontally, in a styrofoam box, $4 \mathrm{~cm}$ above the surface of liquid nitrogen for $10 \mathrm{~min}$ before being plunged into it ("rapid freezing"). The other half of the straws were placed vertically in the freezing chamber of a biological freezer (CryoLogic, Australia) programmed to lower the temperature at a rate of $0.5^{\circ} \mathrm{C} \cdot \mathrm{min}^{-1}$ between $5^{\circ} \mathrm{C}$ and $-10^{\circ} \mathrm{C}$ and of $8{ }^{\circ} \mathrm{C} \cdot \mathrm{min}^{-1}$ between $-10^{\circ} \mathrm{C}$ and $-60^{\circ} \mathrm{C}$, followed by immersion into liquid nitrogen ("slow freezing").

After an average storage of one month, the straws were thawed in a water-bath at $37{ }^{\circ} \mathrm{C}$ for $1 \mathrm{~min}$. Thawed semen was diluted 1:2 with a TRIS buffer pre-warmed to $37^{\circ} \mathrm{C}$ [4] and subjected to evaluation. Two straws were thawed for each ejaculate and treatment: on one straw subjective motility, plasma membrane integrity and chromatin denaturation were evaluated, while the other straw was used for the computerised sperm motility analyses.

\subsection{Post-thaw semen evaluation}

\subsubsection{Motility}

Thawed semen was evaluated subjectively for progressive motility under a phase contrast microscope equipped with a $38^{\circ} \mathrm{C}$ heated stage and $400 \times$ magnification. The observation was done by two operators. Each sample was evaluated at hour 0 and each hour during $8 \mathrm{~h}$ of incubation at $38{ }^{\circ} \mathrm{C}$.

Motility was also assessed by the CEROS 12.1 M Analyser (Hamilton-Thorne Research, Beverly, USA) immediately after thawing and after $2 \mathrm{~h}$ of incubation at $37^{\circ} \mathrm{C}$. Two $5 \mu \mathrm{L}$ drops of frozen-thawed and diluted semen were placed in a Cell-Vu chamber (Millennium Sciences Inc. New York, USA) and 8 fields were analysed for each drop. The settings of the instrument were as fol- lows: temperature $37^{\circ} \mathrm{C}$; frames acquired 30 ; Frame rate $60 \mathrm{~Hz}$; Minimum contrast 70; Straightness threshold $80 \%$; Low VAP cutoff 20; Medium VAP cut-off 40 . The motility parameters obtained by the CEROS analyser were total motility (\%), progressive motility (\%), VSL (straight line velocity, $\mu \mathrm{m} \cdot \mathrm{s}^{-1}$ ), VAP (average path velocity, $\mu \mathrm{m} \cdot \mathrm{s}^{-1}$ ), VCL (curvilinear line velocity, $\mu \mathrm{m} \cdot \mathrm{s}^{-1}$ ), ALH (amplitude of lateral head displacement, $\mu \mathrm{m}$ ), $\mathrm{BCF}$ (beat cross frequency, $\mathrm{Hz}$ ), STR (straightness, \%), LIN (linearity, \%).

\subsubsection{Sperm membrane function: HOS test}

Immediately after thawing, $100 \mu \mathrm{L}$ of diluted semen were added to $900 \mu \mathrm{L}$ of a hypo-osmotic (60 mOsm) fructose solution [12] and incubated at $37{ }^{\circ} \mathrm{C}$ for $45 \mathrm{~min}$. Micro-drops of the sperm suspension were placed on a pre-warmed slide, covered with a coverslip, and observed under a phase contrast microscope at $400 \times$ magnification. For each sample at least 100 spermatozoa were observed by each of two operators classifying them as HOS positive (HOS+), when spermatozoa showed a swelled and coiled tail.

\subsubsection{Chromatin integrity: AO test}

Just after thawing, small aliquots of diluted semen were smeared on slides and air dried. The slides were fixed in Carnoy solution (3:1 methanol: glacial acetic acid) for $6 \mathrm{~h}$, then covered with $0.1 \%$ AO staining solution, according to the method of Tejada et al. [14]. After $5 \mathrm{~min}$, the slides were washed with tap water, covered with a coverslip and immediately sealed with nail polish. The slides were examined in a fluorescent microscope (Olympus Italia, Milan, Italy), using a 490-nm excitation filter and a 530-nm barrier filter, with at least 100 spermatozoa observed per slide (two slides for each ejaculate and each treatment). Spermatozoa carrying a normal DNA appear as green heads $(\mathrm{AO}+)$, while single-stranded denaturated DNA produces a yellow-orange fluorescence. 


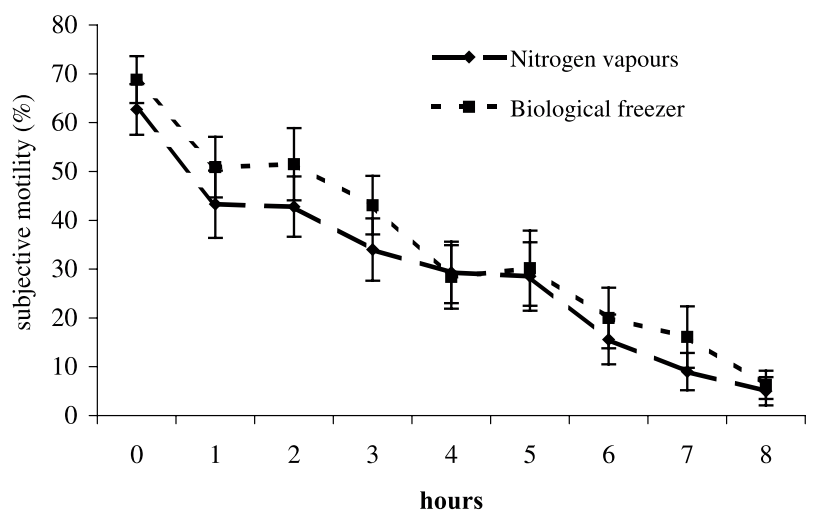

Figure 1. Post-thaw decline of subjective motility (mean value \pm SEM) of dog semen frozen over nitrogen vapours or in a biological freezer $\left(8 \mathrm{~h}\right.$ of incubation at $\left.38^{\circ} \mathrm{C}\right)$.

\subsubsection{Statistical analysis}

To evaluate the effect of different factors and their interactions on the variables considered in the study, data were subjected to the analysis of variance using the General Linear Model univariate procedure of the SPSS software (SPSS INC., Chicago). Percentage values were submitted to angular transformation before analysis.

Subjective motility, total and progressive motility and all the motility parameters read by the CEROS analyser, and percentage of $\mathrm{AO}+$ spermatozoa, immediately after thawing, were analysed taking into account the effect of the freezing procedure, dog and their interaction; all the parameters read by the CEROS analyser were further analysed in order to also evaluate the differences between the values recorded immediately at thawing and after $2 \mathrm{~h}$ of incubation.

Subjective motility over time (from thawing to hour 8) was analysed considering the effects of the freezing procedure, dog and their interaction, with time (hour 0 to hour 8) as the covariate.

The percentage of HOS+ spermatozoa was analysed considering the effects of the freezing procedure, dog, operator and their interactions.
The correlation between subjective motility, total and progressive motility, HOS+ results from the two operators, and $\mathrm{AO}+$ spermatozoa, was assessed by calculation of the Pearson correlation coefficients.

The results were considered statistically significant when $P<0.05$.

\section{RESULTS}

Immediately after thawing as well as in any of the subsequent single time points, subjective motility was comparable in the two treatments; however the slopes of the two curves were significantly different $(\mathrm{F}=$ $4.38 ; P=0.04)$, with a slower decline for the curve referred to the biological freezer (Fig. 1 and Tab. I). Also the effect of dog and the interaction between dog and treatment were significant over time $(\mathrm{F}=7.82$; $P<0.01$ and $\mathrm{F}=3.45 ; P<0.01$ respectively).

Total and progressive motility and kinetic characteristics of thawed spermatozoa, measured by the CEROS analyser immediately post-thaw and after $2 \mathrm{~h}$ of incubation, are shown in Table II. At hour 0, total motility was significantly higher in the samples frozen in the biological freezer while progressive motility, although higher, was not 
Table I. Subjective motility (mean value \pm SEM) immediately after thawing and hourly, over $8 \mathrm{~h}$ of incubation at $38^{\circ} \mathrm{C}$.

\begin{tabular}{lcccccccccc}
\hline & Hour & 0 & 1 & 2 & 3 & 4 & 5 & 6 & 7 & 8 \\
\hline Nitrogen & Mean & 62.7 & 43.3 & 42.8 & 34.0 & 29.3 & 28.5 & 15.6 & 9.0 & 5.0 \\
vapours & SEM & 5.2 & 6.9 & 6.2 & 6.4 & 6.3 & 7.0 & 5.1 & 3.8 & 2.9 \\
Biological & Mean & 68.8 & 50.9 & 51.5 & 43.1 & 28.4 & 30.2 & 20.0 & 16.1 & 6.3 \\
freezer & SEM & 4.8 & 6.2 & 7.4 & 6.0 & 6.5 & 7.7 & 6.2 & 6.3 & 2.9 \\
\hline
\end{tabular}

Table II. Motility parameters measured by the CEROS analyser at thawing and after $2 \mathrm{~h}$ of incubation (mean values $\pm \mathrm{SEM}) . \mathrm{TM}=$ total motility, $\mathrm{PM}=$ progressive motility, $\mathrm{VAP}=$ velocity average pathway, $\mathrm{VSL}=$ velocity straight line, $\mathrm{VCL}=$ curvilinear velocity, $\mathrm{ALH}=$ amplitude lateral head, $\mathrm{BCF}$ $=$ beat cross frequency, $\mathrm{STR}=$ straightness, $\mathrm{LIN}=$ linearity.

\begin{tabular}{|c|c|c|c|c|c|c|c|c|c|}
\hline & $\mathrm{TM}$ & PM & VAP & VSL & VCL & ALH & $\mathrm{BCF}$ & STR & LIN \\
\hline \multicolumn{10}{|l|}{ Hour 0} \\
\hline Vapours & $50.2 \pm 6.0^{\mathrm{a}}$ & $41.4 \pm 5.1$ & $118.8 \pm 2.9^{\mathrm{a}}$ & $110.5 \pm 2.8^{\mathrm{a}}$ & $155.7 \pm 3.3^{\mathrm{A}}$ & $5.3 \pm 0.2^{\mathrm{a}}$ & $27.7 \pm 0.8$ & $91.5 \pm 0.5$ & $71.0 \pm 1.3$ \\
\hline Freezer & $64.8 \pm 3.4^{b}$ & $51.1 \pm 3.2$ & $103.1 \pm 3.7^{b}$ & $94.9 \pm 3.6^{\mathrm{b}}$ & $136.7 \pm 4.9^{\mathrm{B}}$ & $5.0 \pm 0.2^{b}$ & $25.6 \pm 0.6$ & $90.5 \pm 0.8$ & $70.0 \pm 1.6$ \\
\hline \multicolumn{10}{|l|}{ Hour 2} \\
\hline Vapours & $30.3 \pm 5.7^{c}$ & $16.7 \pm 3.9$ & $105.8 \pm 6.3^{\mathrm{c}}$ & $86.0 \pm 5.7^{\mathrm{c}}$ & $185.1 \pm 8.9^{C}$ & $8.3 \pm 0.3^{c}$ & $22.4 \pm 1.0$ & $80.0 \pm 1.0$ & $48.3 \pm 1.5$ \\
\hline Freezer & $36.3 \pm 4.8^{\mathrm{d}}$ & $19.9 \pm 3.3$ & $92.3 \pm 5.0^{\mathrm{d}}$ & $75.4 \pm 4.4^{\mathrm{d}}$ & $157.0 \pm 8.2^{\mathrm{D}}$ & $7.4 \pm 0.3^{\mathrm{d}}$ & $23.4 \pm 1.2$ & $81.2 \pm 0.7$ & $50.6 \pm 1.3$ \\
\hline
\end{tabular}

Mean values in a column that differ significantly $(P<0.05)$ have different small letters; if $P<0.01$, superscript letters are capitals.

Table III. Percentage of HOS positive spermatozoa evaluated by two operators at thawing (mean values \pm SEM).

\begin{tabular}{lcc}
\hline & HOS $+(\%)$ operator I & HOS+ $(\%)$ operator II \\
\hline Nitrogen vapours & $46.5 \pm 4.0^{\mathrm{A}}$ & $52.5 \pm 4.5^{\mathrm{A}}$ \\
Biological freezer & $60.8 \pm 2.8^{\mathrm{B}}$ & $65.3 \pm 2.1^{\mathrm{B}}$ \\
\hline
\end{tabular}

Superscript different letters in a column indicate a significant level $P=0.01$.

statistically different $(P=0.05)$. On the contrary, VAP, VSL, VCL and ALH were significantly higher in the samples frozen over nitrogen vapours. The BCF, STR and LIN values did not differ between the two freezing procedures. All parameters, except VCL and ALH, showed a significant decrease after $2 \mathrm{~h}$ of incubation and the differences between freezing procedures were confirmed. Total motility remained significantly higher $(P=0.01)$ in the samples frozen in the biological freezer, while progressive motility was not different between the treatments. For the other parameters, the same trend shown at hour 0 was observed at hour 2 . A significant effect of individual dogs was observed for all motility parameters, with the exception of STR and LIN.

Sperm membrane function was significantly influenced by the freezing procedure, dog and their interaction, but not by the operator; the proportion of spermatozoa showing a functional plasma membrane was significantly higher in the samples frozen in the biological freezer $(P=0.01)$ (Tab. III).

Neither the freezing procedure nor the dog had a significant effect on AO staining 
Table IV. Pearson's correlation coefficients between the variables considered in the study.

\begin{tabular}{lccccc}
\hline & $\begin{array}{c}\text { Subjective } \\
\text { motility }\end{array}$ & HOS+ (I) & HOS+ (II) & AO+ & $\begin{array}{c}\text { Progressive motility } \\
\text { (analyser) }\end{array}$ \\
\hline HOS+ (I) & 0.08 & & & & \\
HOS+ (II) & 0.22 & $0.83^{* *}$ & & & \\
AO+ & 0.32 & -0.02 & 0.17 & & \\
Progressive motility (analyser) & $0.65^{* *}$ & 0.38 & $0.43^{*}$ & 0.25 & \\
Total motility (analyser) & $0.66^{* *}$ & $0.35^{*}$ & $0.41^{*}$ & 0.31 & $0.98^{* *}$ \\
\hline
\end{tabular}

$* P<0.05$. ** $P<0.001$

patterns. The proportion of spermatozoa showing single stranded, denaturated DNA was always low, corresponding to $2.5 \% \pm$ $0.9 \%$ in samples frozen over nitrogen vapours and $6.0 \% \pm 3.5 \%$ in samples frozen in the biological freezer.

The Pearson correlation coefficients showed that there was a significant correlation between subjective motility and motility measured by the analyser, between the proportion of HOS+ spermatozoa and total and progressive motility, and between the HOS results obtained by the two operators (Tab. IV). AO results were not correlated with any parameter.

\section{DISCUSSION}

The use of biological freezers for dog semen has been rather limited, and straw freezing is usually achieved either by placing the straws horizontally at different distances from the surface of liquid nitrogen or by lowering the straws inside a nitrogen tank in different steps $[6,18,19]$. To date, the most appropriate velocity of temperature descent during the process of canine semen preservation has not been determined. The results of different studies are difficult to compare because experimental conditions are never the same, differing in the composition of extenders, steps of glycerol addition, final cryoprotectant concentration or thawing time-temperature combination $[3,6,20-22]$.
When the same extender and semen handling than those used in this study were employed, the results were not conclusive. A slow freezing rate $\left(3^{\circ} \mathrm{C} \cdot \mathrm{min}^{-1}\right.$ between 4 and $-6{ }^{\circ} \mathrm{C} ; 10^{\circ} \mathrm{C} \cdot \mathrm{min}^{-1}$ between -6 and $-40{ }^{\circ} \mathrm{C}$; $50{ }^{\circ} \mathrm{C} \cdot \mathrm{min}^{-1}$ between -40 and $-140{ }^{\circ} \mathrm{C}$ ) negatively affected spermatozoal motility compared to a faster rate $\left(3^{\circ} \mathrm{C} \cdot \mathrm{min}^{-1}\right.$ between 4 and $-6{ }^{\circ} \mathrm{C} ; 50{ }^{\circ} \mathrm{C} \cdot \mathrm{min}^{-1}$ between -6 and $-140{ }^{\circ} \mathrm{C}$ ) [21]. However, a slow rate tended to increase the percentage of spermatozoa showing an intact plasma membrane. In a previous experiment [6], when fast freezing, obtained by placing the straws at a distance of $4 \mathrm{~cm}$ from liquid nitrogen was compared to a slower freezing rate obtained by step-lowering the straws in a nitrogen tank, spermatozoal motility was not higher immediately post-thaw, but was maintained longer during incubation at $38{ }^{\circ} \mathrm{C}$. No effect on plasma membrane and acrosome integrity was observed [6]. In our study, the slow freezing rate was advantageous compared to the fast one, both in terms of post-thaw sperm motility and plasma membrane function. However, sperm velocities (VAP, VSL, VCL) and lateral head displacement (ALH) were higher in semen samples frozen with the fast rate. How these parameters may influence fertility in the canine species remains to be determined.

An advantage of slower freezing rates was reported by several other authors, albeit using different freezing extenders and/or straws of different volumes, factors that can interact with freezing rates, thus affecting 
semen quality at thawing. For example, using $0.25 \mathrm{~mL}$ straws, the slower rate obtained $10 \mathrm{~cm}$ above liquid nitrogen gave significantly higher post-thaw motility and HOS positive spermatozoa than when the straws were placed at only $5 \mathrm{~cm}$ from liquid nitrogen [22]. Also using epididymal spermatozoa, a lower freezing rate $\left(3-11^{\circ} \mathrm{C} \cdot \mathrm{min}^{-1}\right)$ increased motility and the proportion of intact plasma membranes at thawing [3]. Another study showed that a moderate freezing rate $\left(-5^{\circ} \mathrm{C} \cdot \mathrm{min}^{-1}\right.$ from 5 to -15 and $-20^{\circ} \mathrm{C} \cdot \mathrm{min}^{-1}$ from -15 to $-100^{\circ} \mathrm{C}$ ) was better in preserving spermatozoal motility after thawing than both a slow $\left(-2{ }^{\circ} \mathrm{C} \cdot \mathrm{min}^{-1}\right.$ from 5 to -15 and $-10{ }^{\circ} \mathrm{C} \cdot \mathrm{min}^{-1}$ from -15 to $\left.-100{ }^{\circ} \mathrm{C}\right)$ and a fast rate $\left(-75^{\circ} \mathrm{C} \cdot \mathrm{min}^{-1}\right)$ [20]. The freezing rate we adopted in the biological freezer was lower than most of those previously described, and semen subjected to controlled freezing showed a higher post-thaw motility, which decayed with a significantly different slope as compared to fast freezing over nitrogen vapours. The other motility parameters read by the CEROS analyser showed that slowly frozen spermatozoa had a lower velocity (VAP and VSL). The significance of this result is not clear, although potentially negative, since significant positive correlations were observed between VSL, VAP and fertility in other species [23]; spermatozoa frozen in the biological freezer also exhibited lower lateral head displacement and curvilinear velocity. These parameters increase during the capacitation process and a precocious capacitation could lead to a premature acrosome reaction and then reduce the life span and fertility of spermatozoa [24]. Capacitation-like changes can be induced by semen preservation and by the media in contact with the spermatozoa [25-26]; and the increase in ALH observed $2 \mathrm{~h}$ after thawing could be an expression of this phenomenon.

This study indicates that the subjective evaluation of immediate post-thaw motility alone might not be a sensitive enough parameter to pick differences that can be better evaluated by other methods, such as repeated measures of motility over time, computerised motility analyses or the evaluation of plasma membrane integrity, which seems to be a more sensitive parameter. The HOS test, employed immediately post-thaw, was correlated to total and progressive motility evaluated by CEROS at hour 0 and highlighted a difference between treatments that was not evident at the subjective estimation of motility immediately post-thaw. However, plasma membrane integrity estimation over time would have allowed to have more information on the samples, such as if the immotile spermatozoa seen after several hours of incubation were still viable.

Subjective estimation always resulted in a mean higher motility as compared to the CEROS motility analyser. However the two methods were significantly correlated and any potential over/under estimation would equally affect all the samples.

To our knowledge, this was the first study where chromatin denaturation was evaluated on frozen-thawed canine spermatozoa by A/O staining. The freezing procedures employed here did not cause DNA injuries in a high proportion of spermatozoa, since more than $90 \%$ of them showed intact DNA. Previous works on sperm from bulls, stallions and boars, established a threshold value of DNA denaturation $(30 \%$ or more) that is predictive of sub/infertility [16]. Under our conditions, the AO test was not useful to detect significant differences between treatments. This was in contrast with the findings of Hammadeh et al. [27], who found a significantly higher percentage of intact DNA in slowly frozen human spermatozoa, but is in agreement with a previous work on stallion semen, where very low percentages of denaturated DNA were found both in fresh and frozen samples [28]. A different susceptibility to denaturation may be related to species differences in sperm chromatin structure. In man, sperm chromatin is very variable, ranging from a very condensed structure to a more granular appearance, with vacuoles of various sizes, which might be indicative of immaturity. 
Also the protamine content of human sperm nuclei, lower than in bulls and stallions, and the presence of a second type of protamine, with less free-sulphydryl groups available for disulfide bonds, leads to a less compact structure more susceptible to alteration in this species [29].

All parameters were affected by the dog factor, indicating that different dogs may have different sensitivities to the freezing procedures, as already pointed out in previous works [3, 19].

In conclusion, although the number of dogs included in this study was limited, a slow freezing rate obtained with a biological freezer was advantageous, compared to freezing at a faster rate over the liquid nitrogen surface, in terms of spermatozoa motility, longevity and functionality of plasma membrane after thawing. Sperm velocities, however, were lower when using the biological freezer, and no difference between treatments in DNA denaturation was observed. Further studies are needed to evaluate the fertility of spermatozoa frozen-thawed with these methods.

\section{ACKNOWLEDGEMENTS}

The authors thank Paolo Zucchini for his precious technical assistance.

\section{REFERENCES}

[1] Mazur P. Freezing of living cells: mechanisms and implications. Am J Physiol 1984, 247: 125-142.

[2] Hammadeh ME, Szarvasy D, Zeginiadou T, Rosenbaum P, Georg T, Schmidt W. Evaluation of cryoinjury of spermatozoa after slow (programmed biological freezer) or rapid (liquid nitrogen vapour) freeze-thawing techniques. J Assist Reprod Genet 2001, 18: 364 370.

[3] Yu I, Songsasen N, Godke RA, Leibo SP. Differences among dogs in response of their spermatozoa to cryopreservation using various cooling and warming rates. Cryobiology 2002, 44: 62-78.
[4] Rota A, Ström B, Linde-Forsberg C, RodriguezMartinez H. Effects of Equex STM Paste on viability of frozen-thawed dog spermatozoa during in vitro incubation at $38^{\circ} \mathrm{C}$. Theriogenology 1997, 47: 1093-1101.

[5] Burgess CM, Bredl JC, Plummer JM, England GC. Vital and ultrastructural changes in dog spermatozoa during cryopreservation. J Reprod Fertil Suppl 2001, 57: 357-363.

[6] Peña A, Linde-Forsberg C. Effects of Equex, one- or two-step dilution, and two freezing and thawing rates on post-thaw survival of dog spermatozoa. Theriogenology 2000, 54: 859-875.

[7] Neild D, Chaves G, Flores M, Mora N, Beconi $\mathrm{M}$, Aguero A. Hypoosmotic test in equine spermatozoa. Theriogenology 1999, 51: 721727.

[8] Jeyendran RS, Van der Vent HH, PerezPelaez M, Crabo BG, Zaneveld LJD. Development of an assay to assess the functional integrity of human sperm membrane and its relationship to other semen characteristics. J Reprod Fertil 1984, 70: 219-228.

[9] Rota A, Penzo N, Vincenti L, Mantovani R. Hypo-osmotic Swelling (HOS) as a screening assay for testing in vitro fertility of bovine spermatozoa. Theriogenology 2000, 53: 14151420 .

[10] Correa JR, Heersche G, Zavos PM. Sperm membrane functional integrity and response of frozen-thawed bovine spermatozoa during the hypoosmotic swelling test incubation at varying temperatures. Theriogenology 1997, 47: 715-721.

[11] England GCW, Plummer JM. Hypo-osmotic swelling of dog spermatozoa. J Reprod Fertil Suppl 1993, 47: 261-270.

[12] Kumi-Diaka J. Subjecting canine semen to the hypo-osmotic test. Theriogenology 1993, 39: 1279-1289.

[13] Royere D, Hamamah S, Nicolle JC, Barthelemy C, Lansac J. Freezing and thawing alter chromatine stability of ejaculated human spermatozoa: fluorescence acridine orange staining and Feulgen-DNA cytophotometric studies. Gamete Res 1988, 21: 51-57.

[14] Tejada RI, Mitchell JC, Norman A, Marik JJ, Friedman S. A test for practical evaluation of male fertility by acridine orange (AO) fluorescence. Fertil Steril 1984, 42: 87-91.

[15] Evenson DP, Darzynkiewicz Z, Melamed MR. Relation of mammalian sperm chromatin heterogeneity to fertility. Science 1980, 240: 1131-1133. 
[16] Evenson DP, Jost L. Sperm chromatin structure assay is useful for fertility assessment. Methods Cell Sci 2000, 22: 169-189.

[17] Penfold LM, Jost L, Evenson DP, Wildt DE. Normospermic versus teratospermic domestic cat sperm chromatin integrity evaluated by flow cytometry and intracytoplasmic sperm injection. Biol Reprod 2003, 69: 1730-1735.

[18] Smith FO. Cryopreservation of canine semen, technique and performance. $\mathrm{PhD}$ thesis, University of Minnesota (USA), 1984.

[19] Dobrinsky I, Lulai C, Barth AD, Post K. Effects of four different extenders and three different freezing rates on post-thaw viability of dog semen. J Reprod Fertil Suppl 1993, 47: 291-296.

[20] Olar TT, Bowen RA, Pickett BW. Influence of extender, cryopreservative and seminal processing procedures on postthaw motility of canine spermatozoa frozen in straws. Theriogenology 1989, 31: 451-461.

[21] Rota A, Linde-Forsberg C, Vannozzi J, Romagnoli S, Rodriguez-Martinez H. Cryosurvival of dog spermatozoa at different glycerol concentrations and freezing/thawing rates. Reprod Dom Anim 1998, 33: 355-361.

[22] Mohanachandran SR, Kalatharan J, Rajasekaran J. Effect of cryopreservation on the viability and membrane integrity of canine spermatozoa. Indian J Anim Reprod 1999, 20: 142145.

[23] Wilhelm KM, Graham JK, Squires EL. Comparison of the fertility of cryopreserved stallion spermatozoa with sperm motion analyses, flow cytometric evaluation, and zona-free hamster oocyte penetration. Theriogenology 1996, 46: 559-578.

[24] Watson PF. Recent developments and concepts in the cryopreservation of spermatozoa and assessment of their post-thawing function. Reprod Fertil Dev 1995, 871-891.

[25] Rota A, Peña A, Linde-Forsberg C, RodriguezMartinez $\mathrm{H}$. In vitro capacitation of fresh, chilled and frozen-thawed dog spermatozoa assessed by the chlortetracycline assay and changes in motility patterns. Anim Reprod Sci 1999, 57: 199-215.

[26] Iguer-Ouada M, Verstegen JP. Evaluation of the Hamilton Thorne Computer-based Automated System for dog semen analysis. Theriogenology 2001, 55: 733-749.

[27] Hammadeh ME, Greiner S, Rosenbaum P, Schmidt W. Comparison between Human Sperm Preservation Medium and TEST-yolk buffer on protecting chromatin and morphology integrity of human spermatozoa in fertile and subfertile men after freeze-thawing procedure. J Androl 2001, 22: 1012-1018.

[28] Blottner S, Warnke C, Tuchscherer A, Heinen $\mathrm{V}$, Torner $\mathrm{H}$. Morphological and functional changes of stallion spermatozoa after cryopreservation during breeding and non breeding season. Anim Reprod Sci 2001, 65: 75-88.

[29] Evenson DP, Larson K, Jost L. Sperm chromatin structure assay: its clinical use for detecting sperm DNA fragmentation in male infertility and comparison with other techniques. J Androl 2002, 23: 25-43. 